\title{
Proceedings of the meeting of the Association of British Neurologists, London, 28-30 September 1989
}

CAN THE BRAIN BEAT TIME IN PARKINSON'S DISEASE?

CD Ward. University of Southampton, Southampton

Several deficits in Parkinson's Disease (PD) might be based on a failure of internal pacing. A minimum criterion for internal pacing is the ability to produce a regular beat at a given mean frequency, without external pacing. Control of regular rhythms was studied in seven patients with PD and seven matched controls using a touch sensitive board. Trains of tones were emitted at five test frequencies from 5 to $0.3 \mathrm{~Hz}$, giving inter-tone-intervals of $0 \cdot 2,0 \cdot 4,0 \cdot 8,1 \cdot 6$ and $3 \cdot 2$ seconds. Subjects were required to continue tapping at the test frequency immediately after the tones ceased, or after unfilled intervals of 30 to 300 seconds. Mean inter-response interval (RI) did not differ between the groups at any frequency. $\mathrm{RI}$ variance was higher in PD, confirming previous studies. Both groups consistently tapped too fast at the lowest frequency. Patients did not fatigue during sustained tapping in a free tapping task. Performance was not impaired after delays during which some form of mental rehearsal presumably occurred. Most parameters of the putative internal pacer were thus shown to be normal. Data did not support the two-stage model for the maintenance of mean RI at different tapping rates, but conformed to an autoregressive model in both PD and controls.

THE ROLE OF URETHRAL SPHINCTER ELECTROMYOGRAPHY IN THE DIFFERENTIAL DIAGNOSIS OF PARKINSONISM

I Eardley, NP Quinn, CJ Fowler, RA Kirby, CD Marsden, R Bannister. St Bartholomew's and the National Hospitals for Nervous Diseases, London

It is now recognised that a significant proportion of patients who present with parkinsonism, actually suffer from multiple system atrophy (MSA). Differential diagnosis from idiopathic Parkinson's disease (PD) may be difficult, particularly in the early stages of the disease. In previous studies, we have shown that patients with MSA presenting with autonomic failure have abnormal urethral sphincter electromyography (EMG) activity, whilst in patients with PD this EMG activity is normal.

We performed urethral sphincter EMG with individual motor unit analysis on 54 patients presenting with parkinsonism and correlated the findings with their subsequent clinical course and diagnosis.

In 26 patients, a clinical diagnosis of probable/definite MSA was made and of these, 16 had abnormal urethral sphincter EMG. In 13 patients a diagnosis of probable/ definite PD was made, but in only one patien was the urethral sphincter EMG activity abnormal. In the other 15 patients, the distinction between PD and MSA was less certain and of these patients, 5 had abnormal sphincter EMG activity.

These results confirm that an abnorma urethral sphincter EMG in a patient with parkinsonism is highly suggestive of MSA (specificity $=0.92$ ) but a normal result does not exclude the diagnosis (sensitivity $=0.62$ )

IMMUNOPATHOLOGY OF BRAIN STEM PALE BODIES IN PARKINSON'S DISEASE

GE Dale, PN Leigh, A Probst, BH Anderton St George's Hospital Medical School and the Institute of Psychiatry, London

The presence of Lewy bodies in the substantia nigra is an essential diagnostic feature of Parkinson's disease, along with loss of pigmented cells in this region. Immunocytochemical studies have previously shown that neurofilament and tubulin epitopes, and more recently ubiquitin, are components of Lewy bodies. We are interested in the sequence of events leading to Lewy body formation and neuronal death. We report detailed analysis of neuronal changes in parkinsonian substantia nigra highlighted by immunocytochemical staining with antibodies to phosphorylated neurofilamen (BF10) and ubiquitin. We have demonstrated structures which correspond to inclusions described by other authors as pale, colloid or hyaline bodies. These pale bodies appear to represent a heterogeneous population of inclusions which show a spectrum of staining intensities with anti-ubiquitin and BF10. We suggest that pale bodies may represent a pectrum of changes leading to the eventua formation of the classical Lewy body.

STRIATAL D2 RECEPTOR DENSITY IS INVERSELY PROPORTIONAL TO DOPA UPTAKE IN UNTREATED HEMI-PARKINSON'S DISEASE: A POSITRON EMISSION TOMOGRAPHY STUDY GV Sawle, DJ Brooks, V Ibanez, RS J Frackowiak. MRC Cyclotron Unit, Hammersmith Hospital, London

Experimental destruction of the nigrostriatal pathway in animals is followed by both behavioural supersensitivity and by an increase in the number of striatal dopamine receptors measured in vitro. Post mortem studies of D2 receptors in Parkinson's disease have shown either an increase, no change, or decrease in receptor density. We have studied the nigrostriatal system using positron emission tomography with $18 \mathrm{~F}-6$-fluorodopa to assess presynaptic dopa uptake and storage and $11 \mathrm{C}$-raclopride to assess D2 receptor density. Three drug naive hemiparkinsonian patients and three healthy volunteers have been studied. The hemiparkinsonian patients, who represent early disease, demonstrate reduced uptake of $18 \mathrm{~F}-6-$ fluorodopa into putamen (mean influx constant 0.0054 for patients, 0.0108 for controls) with preservation of uptake into caudate (mean influx constant 0.0096 for patients, 0.0111 for controls). Raclopride studies show an increased volume of distribution within the putamen in patients $(1 \cdot 31)$ vs controls (1.12), again without change in caudate (1.12 in patients, 1.14 in controls).

Taking the six subjects together and correlating asymmetry in dopa uptake and raclopride binding, there is an inverse relationship between dopa influx constant and raclopride volume of distributors within the putamen, but not the caudate. This suggests that the failing striatum responds to loss of dopaminergic input by upregulation of putamen D2 receptor density. This compensatory mechanism might contribute to the preservation of normal function in individuals with pre parkinsonian states.

LEBER'S HEREDITARY OPTIC NEUROPATHY: CLINICAL IMPLICATIONS OF GENETIC HETEROGENEITY AND MITOCHONDRIAL DNA HETEROPLASMY

IJ Holt, DH Miller, AE Harding. Institute of Neurology, London

Analysis of mitochondrial DNA from patients with Leber's hereditary optic neuropathy (LHON) and their relatives showed that a mutation at base pair (bp) 11778 , demonstrated by loss of a recognition site for the restriction endonuclease SfaNI was present in four out of eight families. This mutation was associated with a poor prognosis for visual recovery, whereas four of five affected males without the 11778 bp mutation followed for four years or more had regained useful vision. All but one subject demonstrating the $S$ faNI site loss had a variable mixture of mutant and normal mitochondrial DNA in peripheral blood, and the relative proportions appeared to be correlated with the risk of developing or transmitting LHON. Detection of the $11778 \mathrm{bp}$ mutation is potentially useful in confirming a clinical diagnosis of LHON, particularly in patients who do not have affected relatives.

NEUROLOGICAL ASPECTS OF ENDEMIC CRETINISM

JGL Morris, JP Halpern, SC Boyages, GF Maberly, CJ Eastman. Westmead Hospital, Sydney

Little attention has been paid to the neurological aspects of endemic cretinism, syndrome of mental and physical retardation found in areas of severe iodine deficiency. While endemic cretinism has largely disappeared in Europe, it remains a major public health problem in some underdeveloped countries. Endemic cretins are traditionally divided into two types: myxoedematous, where the features of hypothyroidism predominate, and neurological, where the patients are euthyroid but have mental retardation and spastic diplegia. In this study the neurological features of endemic cretinism are described on the basis of a detailed examination of 104 cretins from Quinghai Province, People's Republic of China, and 35 cretins from Central Java. The main findings were of intellectual impairment, deafness, pyramidal and extrapyramidal signs, primitive reflexes and squint. A characteristic abnormality of gait was observed, much of which could be attributed to laxity and deformity of the joints of the lower limbs. A similar pattern of neurological disturbance was seen, regardless of current thyroid status. The findings support the concept that all cretins undergo, to a greater or lesser extent, a similar 
neurological insult in the mid trimester of intrauterine life due to maternal and fetal hypothyroxinaemia secondary to iodine deficiency.

CLINICAL AND SUBCLINICAL LYME DISEASE IN WESSEX

DE Bateman, EC Guy, NF Lawton. Wessex Neurological Centre, Southampton

In the past three years we have seen 14 patients with neurological Lyme disease. Most have presented with Bannwarth's syndrome. Intense pain due to lumbar or dorsal radiculopathy is common and led to an initial diagnosis of prolapsed intervertebral disc in one patient and a malignant cauda equina lesion in a second. Parenchymal involvement is not a feature of Bannwarth's syndrome, but occurred in four patients; two patients had a cerebellar syndrome, one a myelitis and one a brief encephalitic illness. The CSF examination was helpful in diagnosis, and characteristically showed a lymphocytic pleocytosis with a raised protein and a normal sugar. Furthermore, in two patients antibodies to Borrelia burgdorferi were detected in the CSF before the serology became positive. Patients were initially treated with intravenous penicillin but our recent experience suggests that oral tetracycline is as effective; facial palsy and radicular pain improved dramatically in three patients. We have not seen the tertiary complications described in North America.

We have previously reported a $25^{\circ}{ }_{0}$ prevalence of serum antibodies to Borrelia burgdorferi in 41 forestry workers, without a previous history of neurological Lyme disease. Interestingly one man whose serum was positive has subsequently developed acute neurological Lyme disease, indicating that antibodies do not necessarily confer immunity.

\section{A COMPARISON OF THE HANDLING OF}

NEUROLOGICAL OUTPATIENT REFERRALS BY GENERAL PHYSICIANS AND A NEUROLOGIST T Esmonde, V Patterson. Altnagelvin Hospital, Londonderry and Tyrone County Hospital, Omagh

At some district general hospitals patients with neurological symptoms may be referred by general practitioners to a visiting neurologist but at others there is no such access and they must be referred to general physicians. These two types of patient management have never been compared. A prospective six month survey of new outpatients with neurological symptoms as their primary complaint referred to two district general hospitals was undertaken. In Altnagelvin Hospital (catchment population 140,000), where there is a visiting neurologist without general practitioner access, 103 patients were seen by a general physician on their first visit. In the Tyrone County Hospital (catchment population 56,000$), 39$ were seen by a neurologist. Presenting symptoms, diagnosis investigations, referrals and treatments were examined. The symptoms and eventual diagnoses were comparable. In the Altnagelvin group fewer exact diagnoses were made, more investigations were ordered, more drugs were prescribed, more consultant and paramedica referrals were instituted and fewer patients were discharged after the first visit. This suggests that neurological referrals from general practitioners are dealt with more efficiently by neurologists than by general physicians.
THE NATURAL, HISTORY OF ANTERIOR

ISCHAEMIC OPTIC NEUROPATHY

GV Sawle, CB James, RW Ross Russell. St Thomas' Hospital, London

The aetiology of non-arteritic anterior ischaemic optic neuropathy is presumed to be atheromatous changes within posterior ciliary vessels possibly combined with local anomalies of vascular configuration. There is little evidence regarding the prognosis in patients with this condition, which is thought to be generally benign.

We have studied 71 patients who presented to the Medical Eye Unit at St Thomas' Hospital between 1973 and 1987. Information was extracted from case notes; patients were contacted and reviewed in 1988. Follow up was for a mean of 5.3 years. Eight patients were lost to follow up. Fifty six men and 25 women had presented with this condition, 20 developing bilateral disease. Risk factors at diagnosis were diabetes (5), hypertension (19), ischaemic heart disease (3) or previous stroke (5). Visual acuity was $6 / 18$ or worse in 43 eyes, with no perception of light in 5 and $6 / 6$ or better in 18 . The commonest visual field changes were altitudinal or quadrantic defects, or central scotomas.

During the study period there were 19 deaths, which is significantly more than the expected 8.44 calculated from OPCS data $(\mathrm{p}<0.001)$. Nine died from myocardial infarction (expected 2.67), four from stroke (expected 0.93), four from neoplasia, and one each from mitral valve disease and chronic renal failure.

These findings show that this 'ocular' condition carries a more sinister systemic prognosis than is frequently supposed.

INTER- AND INTRA-OBSERVER VARIATION IN THE ASSESSMENT OF INTRA ARTERIAL DIGITAL SUBTRACTION CAROTID ANGIOGRAPHY; A CLINICALLY IMPORTANT PROBLEM? PAG Sandercock, PRD Humphrey, J Slattery. Western General Hospital, Edinburgh

We examined the nature and extent of interand intra-observer variation in the reporting of intra arterial digital subtraction angiograms. We presented angiographic films of 188 internal carotid arteries from a consecutive series of 99 patients with TIA and minor stroke independently to three radiologists. Each radiologist was asked to measure the maximum percentage stenosis of the internal carotid artery above the bifurcation; the three radiologists were 'blind' to the clinical details of the patients and to the reports given by the other radiologists. The same angiograms were presented again to the same radiologists two years later ('blind' to the previous report). The estimates of stenosis were grouped as: none, $1-24^{\circ}{ }_{0}, 25-49^{\circ} \circ$, $50-74^{\circ}, 75-99^{\circ}$ and occluded. The kappa statistic, which assesses the extent of agreement over and above the effects of chance, between radiologists A \& B, A \& C and $B \& C$ were $0.89,0.87$ and 0.85 respectively. The number of clinically important interobserver disagreements (a difference of 2 or more categories of stenosis, or disagreement about the presence of complete occlusion) for $A$ versus $B, A$ versus $C$ and $B$ versus $C$ were $11(5.9 \%$, $95 \%$ CI 2.5-9.3), 7 $\left(3.7^{\circ}{ }_{0}, 95^{\circ}{ }_{0}\right.$ CI $\left.1.0-6.5\right)$ and $13\left(7.0^{\circ}{ }_{0}, 95^{\circ}\right.$ o CI 3.3-10.7) respectively. The intraobserver variations were, as expected, comparable. Despite the recent improvements brought by new technology, inter- and intra-observer variability is still an important problem which in perhaps $5-10^{\circ}$ of cases may have important clinical consequences.

TRANSCRANIAL DOPPLER SONOGRAPHY IN ACUTE, FIRST EVER HEMISPHERIC CEREBRAL INFARCTION

AR Naylor, RJ Sellar, PAG Sandercock, CP Warlow. Western General Hospital, Edinburgh

We have correlated middle cerebral artery (MCA) velocity blood flow with clinical and CT findings in 18 patients with first ever hemispheric cerebral infarction. MCA velocities were measured using the EME TC2-64B transcranial doppler (TCD).

Eight of nine patients with a total anterior circulation infarction (TACI) had significant TCD abnormality [absent MCA signal (4), reduced ipsilateral MCA velocity (2), MCA stenosis (2)], while one patient had bilateral MCA velocities at the lower limit of normal. Five of six patients with a partial anterior circulation infarction (PACI) had a normal TCD study, while one patient was found to have an absent MCA signal. The TCD study was normal in two of three patients with a lacunar stroke, significant reduction of ipsilateral MCA velocity flow being evident in the third.

TCD abnormalities in each subgroup correlated with the presumed underlying pathophysiology of cerebral infarction; large vessel (internal and middle cerebral artery) occlusion in TACI, MCA branch occlusion in PACI and small vessel disease in lacunar infarction (LACI). These preliminary findings suggest that the clinical-CT classification of TACI, PACI and LACI is valid in pathophysiological terms and that TCD sonography may be a useful method of evaluating and monitoring treatment designed to alleviate major vessel occlusion.

REGIONAL CEREBRAL BLOOD FLOW IN MIGRAINE USING 99M-TC-HMPAO AND SPET PTG Davies, TJ Steiner, DC Costa, BE Jones, R Jewkes, F Clifford Rose. Charing Cross Hospital, London

The importance of vasoconstriction and vasodilatation of cerebral blood vessels in the generation and propagation of acute migraine symptoms is uncertain. Regional cerebral blood flow ( $\mathrm{rCBF}$ ) studies at this time have used, almost exclusively, 133-xenon methodology which has poor spatial resolution. Conflicting interpretations of data have emerged, particularly in classical migraine where cortical areas of decreased and increased blood flow have been reported.

We have used $99 \mathrm{~m}$-Tc-Hexamethylpropyleneamineoxime (99m-Tc-HMPAO) and the Novo 810 SPET scanner to obtain high resolution $\mathrm{rCBF}$ images in patients during $(n=29)$ and between $(n=21)$ attacks of migraine; 14 with acute classical attacks (4 in aura) and 15 acute common migraine. Twenty chronic tension headache sufferers have also been studied.

rCBF images in common migraine appear indistinguishable from those from chronic tension headache patients, both during and between attacks. In a minority of acute classical migraine cases, in aura or headache stage, cortical areas of decreased tracer uptake appropriate to symptoms have been seen. Cortical hyperaemia has not been imaged. 
We conclude that $\mathrm{rCBF}$ changes occur only occasionally in classical migraine and not at all in common migraine. Their significance, when they do occur, is uncertain. It has not been possible to demonstrate a vascular basis for most of the symptoms of migraine using this method of $\mathrm{rCBF}$ imaging.

CEREBRAL VENOUS SINUS THROMBOSIS: NEW CAUSES OF AN OLD SYNDROME RW Ross Russell. St Thomas' Hospital, London

Thrombosis of the cerebral venous sinuses was at one time a common cause of raised intracranial pressure in children following otitic infection. This condition is now rare but recent radiological advances have simplified the recognition of venous sinus occlusion and more cases are being detected. The presenting feature is usually raised intracranial pressure but seizures or focal signs caused by haemorrhagic cerebral infarction may also occur.

A series of six recent patients with cerebral venous thrombosis is described. The underlying diagnosis was often delayed and included an abnormal thrombotic state (two patients), arteriovenous malformation in the posterior fossa, bilateral glomus jugulare tumour, granulomatous disease and cerebral lymphoma.

The natural history of the condition is prolonged but deterioration in vision from the effect of chronically raised intracranial pressure on the optic nerve may require active treatment by lumbo-peritoneal shunting or by optic nerve sheath fenestration.

DO ALZHEIMER'S AND HUNTINGTON'S DISEASE PRODUCE QUALITATIVELY DISTINCT FORMS OF DEMENTIA?

JR Hodges, D Salmon, N Butters. University of California School of Medicine, San Diego

A prospective study compared the performance of 14 patients with dementia of Alzheimer type (DAT, mean age 73.6), 14 patients with Huntington's disease (HD, mean age 52.6) and 14 normal controls (mean age 73.1 ) on a battery of semantic and episodic memory tests. The DAT and HD patients were matched for overall level of dementia using the Mini-Mental State Exam MMSE (mean 24.4 both groups). All subjects were tested on two occasions 12 months apart. The DAT patients were significantly more impaired on measures of delayed verbal and figural episodic memory ( $p<0.05$ ), and in addition showed more rapid decline on tests which depend upon the integrity of semantic knowledge that is, Boston Naming, Number Information, Similarities and Category Fluency (all $\mathrm{p}<0.05$ ). In contrast, the HD patients were significantly worse $(p<0.01)$ and showed more rapid decline on the Letter Fluency Test, reflecting their inability to initiate systematic retrieval strategies. The HD patients were also more impaired than DAT patients on a vocabulary test $(p<0.05)$ and on copying geometric figures $(\mathrm{p}<0.01)$. The observed double dissociations offer compelling evidence that aetiologically distinct forms of dementing illness result in differential patterns of cognitive impairment.
S-ADENOSYLMETHIONINE METABOLISM IN DEPRESSION AND DEMENTIA

T Bottiglieri, P Godfrey, B Toone, MWP Carney, EH Reynolds. King's College Hospital and Northwick Park Hospital, London

$\mathrm{S}$-adenosylmethionine (SAM) is the sole methyl donor of many methylation reactions in the nervous system involving neurotransmitters, amines and polyamines, membrane phospholipids, proteins and nucleoproteins. Studies in Italy, in the United Kingdom by ourselves and in the United States have confirmed that SAM has antidepressant properties especially in patients with endogenous depression.

In 35 patients with severe depression CSF SAM levels (mean $56 \pm 13.8 \mathrm{ng} / \mathrm{ml}$ ) were significantly lower than in a neurological control group $(67 \pm 11.5)(p<0.018)$, but the lowest values were seen in 10 patients with dementia $(38.2 \pm 14.6)(p<0.0001)$. The administration of SAM either intravenously or orally resulted in a significant elevation of CSF SAM indicating that SAM crosses the blood brain barrier. Our observations provide a rational basis for the treatment of some forms of depression with SAM. Preliminary observations suggest that studies of SAM treatment in some forms of dementia, especially Alzheimer's disease, would be worthwhile.

PROGRESSIVE LANGUAGE DISORDERS IN CIRCUMSCRIBED CEREBRAL ATROPHY

JPR Dick, J Snowden, PJ Goulding, B Northen, D Neary. Manchester Royal Infirmary, Manchester

Two forms of primary cerebral atrophy of non-Alzheimer type have been increasingly recognized, dementia of frontal type (DFT) and progressive aphasia. We describe thirteen patients with primary cerebral atrophy who presented with a progressive disturbance of language. They formed three clinically distinct neuropsychological subgroups; four patients retained a purely linguistic impairment, six patients went on to develop an additional disorder of conduct whilst three patients developed an associative agnosia. Spatial function was preserved in all patients. In six patients there was a family history of dementia. In the first group CT scans showed atrophy of the left cerebral hemisphere and single photon emission tomography (SPET) demonstrated a marked reduction in the uptake of tracer throughout the left cerebral hemisphere with relatively normal uptake in the right hemisphere; in the second group there was asymmetric bilateral CT atrophy with corresponding bifrontal abnormalities on SPET, both considerably more marked on the left; in the third group bilateral CT abnormalities were accompanied by more symmetrical anterior defects on SPET. The results suggest an aetiological link between progressive aphasia due to left hemisphere atrophy and DFT in which the frontal or temporal lobes may initially and preferentially atrophy.

\section{COMPARATIVE MONOTHERAPY TRIAL AND} PROGNOSTIC EVALUATION IN NEWLY DIAGNOSED EPILEPSY IN ADULTS AJ Heller, P Chesterman, RDC Elwes, P Crawford, D Chadwick, AL Johnson, EH Reynolds. King's College Hospital, London; Walton Hospital, Liverpool and MRC Biostatistics Unit, Cambridge
Recent studies have confirmed the overall good prognosis for newly diagnosed epilepsy, but differences in efficacy between the major anticonvulsant drugs have not been established. We have undertaken a randomised comparative trial of monotherapy with phenobarbitone, phenytoin, carbamazepine or sodium valproate in 241 previously untreated adult epileptic patients. Patients were stratified according to seizure type and randomised to one of the drugs in a small dose, increasing if necessary to a blood drug level in the top of the "therapeutic" range. The median duration of follow up was 26 months (range 3-90).

The value of monotherapy was confirmed. At two years $82^{\circ}$ " of all patients had achieved a six month remission and $60^{\circ}$ " a one year remission. The prognosis for patients with tonic-clonic seizures was significantly better than those with partial seizures, with or without secondary generalised attacks. For both generalised and partial seizures the number of attacks prior to treatment was the most significant adverse prognostic factor. Other important adverse factors were the occurrence of partial seizures alone, and a neurological or psychiatric handicap. The choice of drug was not a significant factor.

FORAMEN OVALE RECORDINGS IN PREOPERATIVE ASSESSMENT FOR TEMPORAL LOBE SURGERY

D Smith, SJ Howell, A Jenkins, JB Miles, LD Blumhardt, DW Chadwick. Walton Hospital, Liverpool

Eighteen patients with intractable partial seizures who were being considered for temporal lobe surgery had foramen ovale EEG recordings. Electrodes were inserted using fluoroscopic screening with patients under general anaesthesia. Antibiotic prophylaxis was commenced pre-operatively. Electrodes were in situ for 1-9 days, mean 4.0 days Although three patients developed facial haematomas only one recording was stopped prematurely because of facial discomfort and there were no cases of meningitis. Seventy nine seizures were recorded, range $0-12$ per patient, mean 4.38 . In eight patients with a consistent interictal surface EEG focus ictal recordings confirmed the lateralisation of the focus. In four patients with no clear interictal surface lateralisation and two patients with bilateral independent interictal surface foci foramen ovale recordings lateralised the seizure onset to one side. In two patients with bilateral surface foci, seizure onsets were recorded originating from each foramen ovale electrode on different occasions. We conclude that this technique, though invasive, appears to be safe, is technically simple and provides useful lateralising and localising evidence when the surface EEG is unhelpful.

AXONAL NEUROFILAMENT BIOCHEMISTRY IN HEXACARBON NEUROPATHY

$M$ Donaghy, $M$ Coleman. University of Oxford, Oxford and St George's Hospital Medical School, London

Hexacarbon glue solvents, such as $\mathrm{n}-\mathrm{Hexane}$ and 2,5-Hexanedione $(2,5-\mathrm{HD})$ cause peripheral neuropathy by disrupting the cytoskeleton that maintains axonal shape. This is 
reflected by accumulation of disorganised neurofilaments (NF) within distended dista segments of axons, causing giant axonal neuropathy. Reports that NF are abnormally rapidly transported along axons in hexacarbon neuropathy suggest a defect of the normal cross-linkage between NF in the axonal cytoskeleton. Such cross-linking is mediated by phosphorylated forms of NF polypeptide subunits. We have examined the polypeptide subunit composition of NF from spinal cords of rats exposed to $0.5 \% 2,5-\mathrm{HD}$ in drinking water. The content of the subunit NFM, which is involved in NF cross-bridging, was reduced fourfold in NF reassembled from homogenised spinal cords. Furthermore the in vitro phosphorylation of NF subunit polypeptides was reduced after 2,5-HD exposure in vivo. Thus hexacarbon exposure alters NF subunit composition and susceptibility to phosphorylation in a manner likely to reduce physiological cross-linkage between NF. This in turn would allow accelerated axonal transport of NF and their distal accumulation within axons. These results add to the growing evidence that altered NF phosphorylation is caused by toxins interfering with neuronal cytoskeletal organisation.

IS DIABETIC POLYNEUROPATHY A VASCULAR DISEASE?

JL Bradley, RHM King, JG Llewelyn, PK Thomas. Royal Free Hospital School of Medicine, London

The peripheral nerve manifestations that may be related to diabetes mellitus can broadly be divided into (1) focal and multifocal neuropathies and (2) a generalised polyneuropathy that is predominantly sensory and autonomic. There is evidence that the focal neuropathies may have a vascular basis, but the concept that diabetic polyneuropathy may also be related to vascular disease has recently been revived. Part of the evidence for this has been the claim that the number of "closed capillaries" in nerve biopsies is increased in diabetic neuropathy and that basal laminal thickening is increased in comparison with normal control nerves. We have undertaken morphometric studies on a series of sural nerve biopsies from 27 diabetics aged 23-57 (mean 39.8) years. The results have been compared with those from 9 control cases aged 28-35 (mean 44.7) years and from 9 patients with hereditary motor and sensory neuropathy (HMSN) type 1, aged 11-57 (mean 32.7) years. No significant difference was detectable in the luminal area of endoneurial capillaries between the three groups. The area of the capillary basal lamina was similarly increased in the diabetic and HMSN nerves as compared with the controls. Vascular alterations in nerve may therefore be secondary to neuropathy and are not necessarily primary.

IMMUNOGENICITY OF HUMAN RECOMBINANT ACETYLCHOLINE RECEPTOR IN MICE

J Palace, A Vincent, D Beeson, J NewsomDavis. Institute of Molecular Medicine, Oxford

In myasthenia gravis (MG), antibodies to muscle acetylcholine receptor (AChR) bind mainly to its alpha subunit, and their production is believed to require $T$ cell help. To study $T$ cell responses in MG patients and experimental animals, we have generated an essentially full length recombinant human $A C h R$ alpha subunit $(r 37-437)$ and have here investigated its immunogenicity in mice. $T$ cell lines specific for $\mathrm{r} 37-437$ were propagated in vitro and tested with smaller recombinant human AChR alpha subunit fragments and synthetic peptides. Immune sera were tested for reactivity with ${ }^{125} \mathrm{I}-\mathrm{r} 37-437$ and ${ }^{125} \mathrm{I}-\mathrm{a}$ BuTx-AChR by immunoprecipitation. In some strains, $T$ cells recognised epitopes that mapped to the carboxy end of the alpha subunit, and their antibodies cross-reacted with native human AChR, recognising a cytoplasmic region (residues 325-368). By contrast, $T$ cells from one strain ( $\mathrm{SJL}$ ) recognised synthetic peptides $36-56$, representing part of the extracellular portion of the alpha subunit. Their antisera reacted with recombinant (denatured) AChR but not with native human AChR.

Studies of this type, aimed at defining in an animal model $T$ and $B$ cell epitopes and their genetic variability, will be a necessary prerequisite for developing experimental approaches to immunotherapy.

THE EPIDEMIOLOGY OF MOTOR NEURONE DISEASE IN SCOTLAND

RJ Swingler, CP Warlow. Western General Hospital, Edinburgh

Between 1968 and 1987, 6009 patients were discharged from Scottish hospitals with a diagnosis of motor neurone disease (ICD8 code 348 from 1968-78 and ICD9 code 335 from 1979 onwards) and the data were linked to identify 3802 individuals who were classified as having this syndrome for the first time. Two thousand one hundred and fifty three $(57 \%)$ were male and the mean age was 62 years. The discharge rate had risen significantly from 4.13 to $4.52 / 10^{5} /$ year $(\mathrm{r} 0.565$; $\mathrm{p}<0.02$ ) and the highest frequency was observed in 60-79 year olds. Rates were much lower in the 0-19 year age group but the frequency of these juvenile and early adult forms had also increased significantly $(0.23$ to $0.51 / 10^{5} /$ year: $\left.r=0.502 ; p<0.05\right)$ despite the introduction of polio vaccination in 1956.

The postcodes of area of residence were available for $2403(92 \%)$ of the patients discharged for the first time between 1975 and 1987 and these data were used to calculate rates for the 10 regions, 56 standard areas and 1211 postcode sectors of Scotland. The mean rate was $3 \cdot 60 / 10^{5} / \mathrm{yr}$ and there were significant differences with values ranging from $0.92 / 10^{5} /$ year $(95 \%$ CI $0.54-1.47)$ in Kirkcaldy, Fife to $7 \cdot 0 / 10^{5} /$ year $(95 \%$ CI 4.69 $10 \cdot 1$ ) in the Western Islands.

AN ABNORMALITY OF GLYCINE METABOLISM IN ALS PATIENTS

RJM Lane, J de Belleroche, SF Moss, G Horne, R Bandopadhyay, F Clifford Rose. Charing Cross Hospital, London

Amino acids have been implicated in the pathogenesis of motor neuron disease (MND) from a number of lines of evidence. In an earlier study from this laboratory, increased levels of glycine, alanine, threonine, isoleucine and phenylalanine were found in the cerebrospinal fluid (CSF) of patients with MND. An association was found between CSF glycine concentration and disease activity (severity/duration). The relevance of this observation to the patho- genesis of MND is further supported by reported cases of familial spinal cord disease with upper and lower motor neuron signs, secondary to hyperglycinaemia resulting from a defect in conversion of glycine to serine.

We have studied changes in plasma amino acids following oral glycine loading $(0.3 \mathrm{~g} / \mathrm{kg})$ in $17 \mathrm{MND}$ patients and nine controls. There were no differences in initial fasting levels but in patients with amyotrophic lateral sclerosis the clearance of glycine from plasma was significantly slower $(p<0.02)$. Patients with progressive muscular atrophy showed a similar abnormality but the difference was less marked $(p<0.04)$. This defect in glycine clearance was not observed in patients with muscle wasting or spasticity from a variety of other causes. Our results indicate an abnormality of transport or metabolism of glycine in MND patients which may be related to pathogenetic factors in this condition.

TWO DIFFERENT MRI PATTERNS IN PROGRESSIVE MULTIPLE SCLEROSIS

AJ Thompson, AG Kermode, DG McManus, BE Kendall, DPE Kingsley, IF Moseley, WI McDonald. Institute of Neurology, London

Several observations have suggested differences in the pathogenesis of the two forms of progressive multiple sclerosis (MS); that which is progressive from onset (primary progressive- $1^{\circ} \mathrm{P}$ ) and that which succeeds an initially relapsing/remitting course (secondary progressive $-2^{\circ} \mathrm{P}$ ). Using MRI, we have scanned 16 patients with $2^{\circ} \mathrm{P}$ and 13 patients with $1^{\circ} \mathrm{P}$ disease. Six patients from each group were also scanned serially for a six month period: two weekly for three month then monthly. $T_{2}$-weighted MRI of brain and spinal cord was performed (Picker 0.5T, $\mathrm{SE}_{2000 / 60} 5 \mathrm{~mm}$ contiguous slices, $256 \times 256$ image matrix). Gadolinium-DTPA (GdDTPA), was given monthly in a dose of 0.1 $\mathrm{mmol} / \mathrm{kg}$. Scans were examined without knowledge of the patients disease pattern.

There was no significant difference between the two groups at entry or degree of deterioration during the study. Patients with $2^{\circ} \mathrm{P}$ MS had more lesions at outset $(p>0.05)$ and in the $1^{\circ} \mathrm{P}$ group lesions were significantly smaller $(p>0.0001)$. In the serial study 102 new lesions were seen in four of the six $2^{\circ} \mathrm{P}$ patients and $97(95 \%)$ showed enhancement with Gd-DTPA. Only 17 new lesions were seen in three of the six patients with $1^{\circ} \mathrm{P}$ MS and none enhanced. The differences in frequency of new lesions and of enhancement were highly significant $(p>0.01$ and $p>$ 0.001 respectively). The absence of enhancement in $1^{\circ} \mathrm{P}$ MS could be interpreted as evidence that inflammation was either absent or of such limited extent or duration that it was undetectable. These findings suggest important differences in the early stages of development of the lesion between $1^{\circ} \mathrm{P}$ and $2^{\circ} \mathrm{P}$ MS.

ISOLATED NON-COMPRESSIVE MYELOPATHY IN A REGIONAL UNIT: DIAGNOSTIC AND PROGNOSTIC VALUE OF NEUROIMAGING, EVOKED POTENTIALS AND CSF EXAMINATION LD Blumhardt, WI McDonald, P Sandercock, P Moore, A Kermode, L Brock, T Nixon, B Kendall, DPE Kingsley, IF Moseley, J Woodrow, G Findlay. Walton Hospital, Liverpool and Institute of Neurology, London 
A representative cohort of newly presenting cases with an isolated non-compressive myelopathy were identified from 2104 sequential patients having myelography in a regional unit during a 36 month period. Seventy one $\left(15^{\circ}{ }_{0}\right)$ of 473 spinal cases who remained undiagnosed after routine blood tests and myelography had multiple investigations. Magnetic resonance imaging (MRI) revealed two further cases with other unsuspected pathologies, who were then excluded. The remaining 69 patients included $55\left(80^{\circ}{ }_{0}\right)$ with chronic progressive or relapsing disease (mean age 41 years, mean symptom duration 46.5 months) and 14 $\left(20^{\circ}{ }_{0}\right)$ with an acute or subacute myelopathy.

Areas of high signal on MRI were found in the white matter in $74^{\circ}$ o of cases, while computed tomography (CT) scans revealed enhancing lesions in only $4^{\circ}{ }_{0}$. The visual evoked response (VER) was abnormal in $20^{\circ}$. and the auditory evoked response (AER) in $19^{\circ}{ }_{0}$. The cerebrospinal fluid (CSF) contained a raised total protein in $25^{\circ} \circ$, an abnormal cell count in $33^{\circ}{ }_{0}$, an IgG/albumin ratio of more than 0.25 in 425 and oligoclonal bands in $38^{\circ}$.

At follow up the overall risk of developing clinically probable MS within five to 42 months (average follow up 22 months) was $17^{\circ}{ }^{\circ}$. An abnormal MRI scan increased the risk four-fold from $6^{\circ}$, to $24^{\circ}$. Patients developing non-spinal relapses initially had more abnormalities on all tests, but no single test predicted the short term outcome. If all laboratory tests were negative the risk on non-spinal relapses was still $10^{\circ}{ }_{0}$.

\section{A STUDY OF UNITED KINGDOM MULTIPLE} SCLEROSIS MORTALITY RATES OVER A 30 YEAR PERIOD

EW Williams, DR Jones, RO McKeran. Croydon Health Authority and St George's Hospital, London

We have studied United Kingdom mortality data over a 30 year period to examine temporal and geographical variations in mortality, changes in survival, and the relationship between mortality and morbidity trends in patients with multiple sclerosis.

This study has shown an overall decline in mortality throughout the United Kingdom of approximately $25 \%$ over the 30 year period ending in 1983, a reduction in the mortality differential between Scotland on the one hand and England and Wales on the other, and no positive correlation between morbidity and mortality. The overall decline in death rates in females was $24 \%$ and in males $30 \%$ over the 30 years of the survey. The total number of deaths declined by $40 \%$ between 1954-58 and 1979-83 in Scotland compared to an $11 \%$ decline for England and Wales, whereas the improvement in survival over the period of the study was similar for both countries and is unlikely to have contributed to the reduction in the mortality differential.

The present study provides evidence that the differential in mortality between Scotland and England has been declining during the survey period and there is no clear evidence of a latitudinal gradient in mortality south of the Scottish border.

A DOUBLE-BLIND STUDY OF L-THREONINE IN PATIENTS WITH SPASTICITY

K-C Lee, V Patterson. Royal Victoria Hospital, Belfast

The essential amino acid L-Threonine has been used to treat spasticity in several small open studies with reported benefits, possibly by increasing spinal cord glycine. We conducted a double-blind, placebo-controlled, crossover study to investigate the antispasticity effect of L-Threonine. Thirtythree patients, most with multiple scleroris, were treated with $6 \mathrm{~g} / \mathrm{d}$ of L-Threonine and placebo given in two treatment periods in a random order. Each treatment period lasted for two weeks, separated by a washout interval of two weeks. Assessments were carried out before treatment and at the end of each period. Spasticity was measured by the Ashworth Scale. Barthel Index and Kurtzke Scales were used to monitor functional change. We used the sequential approach for our crossover study, and a result in favour of L-Threonine at the chosen level of significance ( $p=0.05$ ) was achieved after thirty-three patients were enrolled. Side effects were infrequent. There was no detectable functional change in patients who showed a decrease in muscle tone. L-Threonine has a definite modest antispastic action. The study suggests that the development of glycinergic drugs may be a useful approach to spasticity treatment.

\section{IS THERE A LINK BETWEEN}

FACIOSCAPULOHUMERAL DYSTROPHY AND SNEDDON'S SYNDROME?

RJ Coleman, DA Ingram, $M$ Swash. The London Hospital, London

We have observed facioscapulohumeral (FSH) dystrophy and Sneddon's syndrome (stroke with livedo reticularis) in the same family. A 45 year old woman was investigated because of cerebrovascular disease. At the age of 30 she suffered a left hemiparesis due to infarction in the right middle cerebral artery territory, as shown by CT. Further transient ischaemic attacks occurred and she developed livedo reticularis, which led to the clinical diagnosis of Sneddon's syndrome. Although there was no clinical evidence of myopathy, EMG (carried out because her 27 year old son had FSH dystrophy) showed widespread myopathic changes. Fluorescein angiography showed an area of vessel leakage at the left disc margin. This patient had three other children, all asymptomatic, of whom one had clinical and EMG evidence of FSH dystrophy.

Abnormal retinal vasculature, assessed by fluorescein angiography, has been reported in 56 out of 75 patients with FSH dystrophy. In Sneddon's syndrome the combination of stroke and livedo reticularis implies abnormal vasculature in both the brain and the skin. The coexistence of these two rare conditions in the same family suggests a common cause, which may be important in understanding the pathogenesis.

INTRATHECAL IgG SYNTHESIS DETECTION USING IEF IN IMMOBILISED PH GRADIENTS AND VISUALISED BY IMMUNOBLOTTING GN Cowdrey, JE Rees. Hurstwood Park Neurological Centre, Haywards Heath

Detection of intrathecal IgG synthesis is most commonly achieved by electrophoresis and is demonstrated by the presence of oligoclonal IgG bands in the CSF.

Various electrophoretic methods differ (a) by the degree of resolution and (b) by the sensitivity.
Separation of oligoclonal IgG bands using high resolution isoelectric focusing (IEF) achieves the greatest detection rate.

In conventional IEF using carrier ampholytes the $\mathrm{pH}$ gradient migrates cathodically during electrophoresis and the most alkaline part of the gradient is lost. This means that the alkaline intrathecal IgG is not well resolved.

A new method has been developed in which the $\mathrm{pH}$ gradient is immobilised onto a polyacrylamide gel matrix. The resolution thus obtained is superior to that of any previous method, especially in the alkaline region $(\mathrm{pH} 7-10)$ and a higher detection rate is achieved.

After IEF, the separated proteins are transferred to a polyvinylidene difluoride membrane (immunoblotting) which is then probed with a variety of location reagents including specific antibodies.

A STUDY OF THE POTENTIAL CLINICAL APPLICATION OF LEVODOPA ENTRAPPED WITHIN LIPOSOMES IN THE TREATMENT OF PARKINSON'S DISEASE

G Firth, M Firth, RO McKeran. Atkinson Morley's Hospital and St George's Hospital Medical School, London and Hurstwood Park Neurological Centre, Haywards Heath

Detailed studies of the factors affecting the incorporation of levodopa into large unilamellar liposomes have enabled us to obtain preparations containing very high concentrations of the entrapped drug. By careful control of the conditions of entrapment it has been possible to produce liposomes which show a sustained release of levodopa in vitro in the presence of gastric and duodenal fluids.

Following ethical committee approval clinical studies have been carried out in six patients with various stages of Parkinson's disease. On separate days, and after an overnight fast levodopa has been given orally or entrapped within liposomes to each patient and blood specimens collected over a period of four hours for estimation of levodopa and three methyl levodopa levels. No evidence of a sustained release of levodopa was demonstrated in these studies although there was some evidence of a more rapid uptake of levodopa that had been entrapped within liposomes. No adverse response to the liposomes was noted.

In view of the good tolerance of these preparations further studies will now be undertaken using lipase-stabilised liposome preparations.

THE HEAT-SHOCK RESPONSE IN MOTOR NEURONE DISEASE (MND)

O Garofalo, PN Leigh, PGE Kennedy, GB Clements, BH Anderton. St George's Hospital Medical School, London

Antibodies to ubiquitin label characteristic inclusions in MND anterior horn cells. Ubiquitin has been implicated in the heatshock or stress response, so we have studied the expression and localization of other heatshock proteins (HSPs) in MND spinal cord from six MND and five control cases, which were immunolabelled with an affinitypurified ubiquitin antibody and with monoclonal antibodies to p57 and 72kd HSPs.

HSP antibodies did not label ubiquitinimmunoreactive neuronal inclusions in 
MND, nor was it possible to distinguish MND and control tissue on the basis of anterior horn cell labelling with HSP72 antibodies. With antibodies to p57, MND anterior horn cells were weakly labelled, whereas controls were negative. In addition, HSP72 antibody revealed numerous round immunoreactive structures in ventral horn neuropil; these were present, although less common, in controls. These studies suggest that cell damage in MND may be associated with a stress response in anterior horn cells and neuropil.

OLIGOCLONAL IgG IN TEAR FLUID OF PATIENTS WITH MS

GE Levin, J Bowler, RO McKeran. St George's Hospital, London

Recent reports have claimed that up to $70^{\circ}$ o of patients with multiple sclerosis have oligoclonal IgG in tear fluid. We have studied 28 patients with clinically definite $M S$ and with positive oligoclonal IgG in the CSF in the 27 patients who had had a lumbar puncture. Oligoclonal IgG was identified by agarose gel isoelectric focusing of 10 or $15 \mu \mathrm{l}$ tears, blotting onto nitro-cellulose, and immunoperoxidase staining. Serum from the MS patients was electrophoresed alongside the tears. Tear IgG was measured on 10 of the MS tear samples by an ELISA technique. Twenty three tear fluid samples were collected from 11 healthy subjects for isoelectric focusing and IgG assay. Approximately 1 to 2 years had elapsed between the lumbar puncture for the oligoclonal studies and the tear collection.

No oligoclonal IgG was detected in any tear sample from the MS patients or from healthy controls. The mean tear IgG was marginally greater in the MS patients $(0.94$ $\mathrm{mg} / \mathrm{l}, \mathrm{SD} 0.34)$ than controls $(0.65 \mathrm{mg} / \mathrm{l}, \mathrm{SD}$ $0.33 ; \mathrm{p}=0.031)$. There was a significant correlation between CSF and tear IgG in the $10 \mathrm{MS}$ patients $(\mathrm{p}=0.014)$.

A COMPARISON OF PERCUTANEOUS AND SURFACE-RECORDED F-WAVE

CHRONODISPERSION VALUES IN 75 HEALTHY OR DAMAGED NERVES

WN Macleod. Ninewells Hospital and Medical School, Dundee

By measuring $\mathrm{F}$-wave latencies in the conventional way with a surface electrode, useful electrodiagnostic information may be concealed. The true $F$-wave latency range may be underestimated by this method as the onsets of the later responses would be obliterated by more rapidly propagated $\mathbf{F}$-waves. Only a small minority (about 1\%) of abductor pollicis brevis (APB) motor units participate in any one surface-recorded F-wave, so a needle electrode with a small recording volume, inserted in the test muscle, might detect the onset of depolarisation in motor units with slower conducting axons.

Data came from 36 healthy and 39 damaged median nerves. Studying APB and using supramaximal wrist stimuli; firstly, F-wave minimum and maximum values were taken from 30 consecutive surface-recorded $F$ waves ( $\geqslant 40 \mu \mathrm{V}$; filters at $20 \mathrm{~Hz}-2 \mathrm{KHz}$ ) and secondly, from $10 \mathrm{~F}$-waves recorded with a needle electrode, each recorded twice for verification (filters at $500 \mathrm{~Hz}-10 \mathrm{KHz}$ ).

In both groups, $\mathrm{F}$ chronodispersion was significantly greater $(p<0.05)$ by needle electrode, with maximal latencies greater $(\leqslant$ $9.3 \mathrm{~ms})$ from needle electrode $(p<0.001)$ and minimal latencies shorter from surface electrode $(\mathrm{p}<0.01$ ).

The onset of F-responses in the muscle fibres of motor units which are not among the fastest conducting units in the pool of motor neurones under test can be recorded percutaneously but not through a surface electrode. Using a needle-electrode, electrodiagnostic sensitivity increases when compared with the conventional method of recording $\mathrm{F}$-wave latencies in routine nerve conduction studies, and this technique can be usefully applied to nerve entrapment syndromes and demyelinating neuropathies.

RECOVERY OF CHROMATIC AND ACHROMATIC CONTRAST SENSITIVITY IN ACUTE OPTIC NEURITIS

JP Reffin, JE Rees, AF Harden. Sussex Eye Hospital, Brighton and Hurstwood Park Neurological Centre, Haywards Heath

Patients presenting with acute optic neuritis were given serial achromatic and chromatic contrast sensitivity tests over the ensuing weeks, using a computer-controlled rasterdisplay based system.

Rate of recovery varied widely between subjects, but little between spatial frequencies within the same subject. There was a consistent relationship between thresholds at different spatial frequencies which was independent of the subject, i.e. the sensitivity function was shape invariant during the early stages of recovery. This function peaks at about 1.3 cycles per degree (cy/deg), falling away rapidly at higher spatial frequencies. In contrast, the average sensitivity functions for both a control group (148 eyes) and the unaffected eyes of the patients, peak at $2 \cdot 2 \mathrm{cy} /$ deg, falling away only gradually to either side.

'Red/Green' and tritanopic colour discrimination sensitivity was also found to be directly proportional to low frequency achromatic contrast sensitivity when expressed in terms of photoreceptor contrast at threshold.

Achromatic contrast sensitivity thresholds were averaged for chronic optic neuritis patients ( 17 eyes) with a residual visual deficit: sensitivity was found also to peak at the lower value of $1.3 \mathrm{cy} / \mathrm{deg}$.

The results suggest that early recovery of function is dominated by the recovery of one or more psychophysical channels with a peak sensitivity at low spatial frequencies.
SOME PATIENTS WITH MULTIPLE SCLEROSIS HAVE A DISORDER OF VITAMIN BI 2 METABOLISM

EH Reynolds, J Linnell, J Faludy, M Lumb, M Laundy, I Chanarin. King's College Hospital, and Westminster Children's Hospital, London

We have studied 10 patients with multiple sclerosis and unusual disorders of vitamin $B_{12}$ metabolism. Seven were female and three were male, age range $25-50$ years. All had evidence of multiple lesions, with a fluctuating course in nine. CSF oligoclonal banding was present in eight out of nine patients examined. Only two patients were anaemic. Nine patients were macrocytic, five (out of six) were megaloblastic. All had serum vitamin $B_{12}$ levels less than $200 \mathrm{pg} / \mathrm{ml}$, with low erythrocyte cobalamin levels. Only two had an abnormal Schilling test. The cause of the vitamin $B_{12}$ deficiency in most was uncertain, but abnormalities of plasma $\mathrm{R}$ binders were found in some. We have also found low erythrocyte cobalamin levels and abnormal $R$ binders in some patients with multiple sclerosis with no other evidence of a vitamin $\mathrm{B}_{12}$ problem. Abnormal cobalamin metabolism may play an etiological role in some patients with multiple sclerosis.

PROGRESSIVE OROFACIAL DYSPRAXIA

ASSOCIATED WITH FRONTAL LOBE

HYPOMETABOLISM

PJ Tyrrell, EK Warrington, RSJ Frackowiak, LJ Findley, MN Rossor. MRC Cyclotron Unit, Hammersmith Hospital, and The National Hospital for Nervous Diseases, London

Progressive cognitive deficits due to focal cortical degeneration have been described presenting with dysphasia, dyspraxia or agnosia, or cortical blindness, which may progress to a generalised dementia. Two patients with progressive orofacial dyspraxia, who subsequently developed more widespread intellectual deficits, are described. In both cases positron emission tomography (PET) scanning revealed bilateral frontal hypometabolism.

Two men, aged 65, with an initial presentation of impaired speech production were studied. Verbal comprehension and other language skills were entirely normal at onset. Symptoms progressed over a number of years, and at the time of scanning, one patient was mute, whilst the other had barely intelligible speech. At this stage, the first patient had developed a severe and global dementia. In the second, there had been generalised deterioration, but on some nonverbal tasks he was still able to perform at the lower limits of normal. Marked orofacial dyspraxia was noted. ${ }^{15} \mathrm{O}$ steady state oxygen scans were performed, to obtain regional values of CMRO2.

Reduction in CMRO2 was most marked in the infero-lateral portions of both frontal lobes, with extension posteriorly. These patients manifest a further presentation of focal cortical degeneration, which has progressed to generalised dementia. The underlying pathology is unknown. 